\title{
21 Data usage by industrial partners
}

Which legal framework must apply if research data already covered by consent are to be used for secondary research and/or by industrial partners (e.g.for quality assurance of implants)?

The secondary use of personal data must be justified under data protection law following the same logic as in the case of primary use. In addition to CDPR, a large number of federal and state laws must also be taken into account in the case of additional research institutions and industrial partners. It is not possible to make a general statement about permissible further processing of personal data by other controllers and/or for other purposes. Depending on the controller and the purpose of the data processing, the applicable data protection law must be identified. Either the right to process data can be directly derived from the law, or the processing can be based on a consent.

If there is already a consent in place for the primary use of data this consent would have to cover also the secondary use of data. This may be a seldom situation as it would be particularly difficult to make sure that the new purpose of the secondary use is covered by the old consent and especially that the new controller is covered by the old consent.

As a rule it can be said that for further scientific research with existing personal data there will often be a legal basis that would allow the processing. In 
Part II of the legal opinions: Detailed Questions on organisational and technical measures

such cases, information on further research must of course be provided according to Article 13, 14 GDPR.

On the other hand, there are usually no laws that permit the processing of health data for quality assurance purposes in commercial enterprises. Exceptions arise in particular for reasons of ensuring high standards of quality and safety of medicinal products and medical devices pursuant to Article 9 para. 2 lit. i) GDPR in conjunction with Section 22 para. 1 No. 1 lit. c) BDSG. It should be noted that there are more specific regulations in the AMG and the MPG, that would mostly cover the relevant cases. Accordingly, Section 22 para. 1 No. 1 lit. c) BDSG is viewed as not having its own relevant use case. ${ }^{75}$ These regulations regularly cover the manufacturer, but not a TTP. A TTP could only be involved as a data processor without the consent of the person concerned.

It will, however, usually be possible to carry out quality assurance of products with anonymous or pseudonymous data. If this is the case, the processing of personal data would be inadmissible anyway, as is clear from the principle of data minimisation. 